\title{
VERÖFFENTLICHUNGEN DER
}

\section{HISTORISCHEN KOMMISSION ZU BERLIN \\ BAND 67}

\author{
BEITRÄGE ZU \\ INFLATION UND WIEDERAUFBAU \\ IN DEUTSCHLAND UND EUROPA \\ 1914-1924
}

Herausgeber

GERALD D. FELDMAN

CARL-LUDWIG HOLTFRERICH

GERHARD A. RITTER

PETER-CHRISTIAN WITT

$B A N D 8$

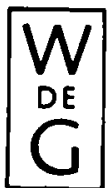

Walter de Gruyter · Berlin - New York 


\title{
THE ADAPTATION TO INFLATION
}

\author{
Edited by \\ GERALD D. FELDMAN \\ CARL-LUDWIG HOLTFRERICH \\ GERHARD A. RITTER \\ PETER-CHRISTIAN WITT
}

\begin{abstract}
With Contributions by
GEROLD AMBROSIUS - DAVID E. BARCLAY - GERALD D. FELDMAN MARTIN GEYER - CARL-LUDWIG HOLTFRERICH · HANS JÜRGEN JAKSCH CLAUS-DIETER KROHN - ANDREAS KUNZ - GUNTHER MAI WILLIAM C. MATHEWS · MERITH NIEHUSS - NORBERT RANFT ROBERT SCHOLZ·STEVEN B. WEBB PETER-CHRISTIAN WITT
\end{abstract}

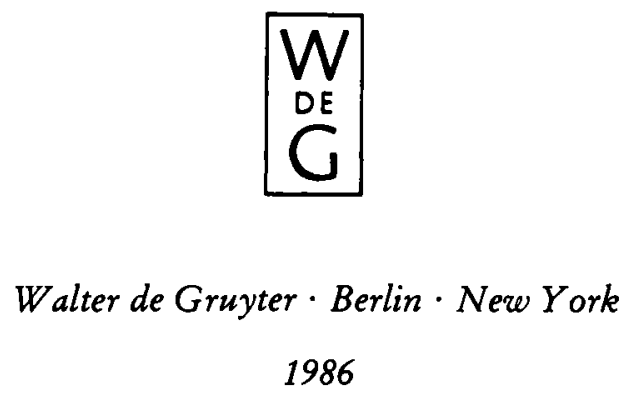




\title{
DIE ANPASSUNG AN DIE INFLATION
}

\author{
Herausgegeben von \\ GERALD D. FELDMAN \\ CARL-LUDWIG HOLTFRERICH \\ GERHARD A. RITTER \\ PETER-CHRISTIAN WITT
}

\begin{abstract}
Mit Beiträgen von
GEROLD AMBROSIUS - DAVID E. BARCLAY - GERALD D. FELDMAN MARTIN GEYER - CARL-LUDWIG HOLTFRERICH - HANS JÜRGEN JAKSCH CLAUS-DIETER KROHN · ANDREAS KUNZ · GUNTHER MAI WILLIAM C. MATHEWS - MERITH NIEHUSS - NORBERT RANFT ROBERT SCHOLZ -STEVEN B. WEBB PETER-CHRISTIAN WITT
\end{abstract}

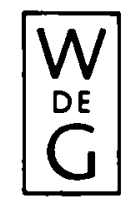

Walter de Gruyter $\cdot$ Berlin $\cdot$ New York 
Die Herausgabe des vorliegenden Bandes erfolgt mit einem Druckzuschuß der Stiftung Volkswagenwerk, Hannover, sowie mit finanzieller Hilfe der Deutschen Bundesbank, Frankfurt am Main

Die Schriftenreihe der Historischèn Kommission zu Berlin erscheint mit Unterstützung des Senators für Wissenschaft und Forschung, Berlin

\section{Lektorat der Schriftenreibe \\ Christian Schädlich}

\section{CIP-Kurztitelaufnabme der Deutschen Bibliothek}

Die Anpassung an die Inflation / hrsg. von Gerald D. Feldman ... Mit Beitr. von Gerold Ambrosius ... - Berlin ; New York : de Gruyter, 1986.

(Veröffentlichungen der Historischen Kommission zu Berlin ; Bd. 67 : Beiträge zu Inflation und Wiederaufbau in Deutschland und Europa 1914-1924; Bd. 8)

Parallelt.: The adaptation to inflation

ISBN 3-11-009935-7

NE: Feldman, Gerald D. [Hrsg.]; Ambrosius, Gerold [Mitverf.]; Historische Kommission , Berlin, West :: Veröffentlichungen der Historischen Kommission zu Berlin / Beiträge zu Inflation und Wiederaufbau in Deutschland und Europa 1914-1924; PT

\section{USA-ISBN 0899252877}

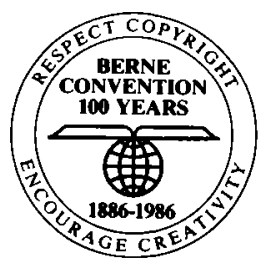

(c)

Copyright 1986 by Walter de Gruyter \& Co., Berlin 30 Printed in Germany

Alle Rechte des Nachdrucks, der photomechanischen Wiedergabe, der Herstellung von Mikrofilmen - auch auszugsweise - vorbehalten

Satz: Historische Kommission zu Berlin, Berlin 38

Druck: Werner Hildebrand, Berlin 65

Einband: Lüderitz \& Bauer, Berlin 61 